\title{
Banking on cord blood stem cells
}

\section{Michael J. Sullivan}

The accuracy and veracity of information provided to parents is crucial to their making an informed choice about whether to bank their child's cord blood; on this point the correspondents on my article (Banking on cord blood stem cells. Nature Rev. Cancer 8, 555-563 (2008)) $)^{1}$, J. J. Nietfeld (Opinions regarding cord blood use need an update. Nature Rev. Cancer 24 Sep 2008 (doi:10.1038/nrg2418-c1)) $)^{2}$ and D. T. Harris (Cord blood stem cells: worth the investment. Nature Rev. Cancer 24 Sep 2008 (doi:10.1038/nrg2418-c2) $)^{3}$, and I are in complete agreement.

Influencing this decision is the likelihood that your child's stored cord blood has a reasonable chance of being used during the time that the cryopreserved cells remain viable $^{1}$.

However, Nietfeld disagrees with most of the previously published opinions that the likelihood of use is indeed minute ${ }^{1}$. In their recent paper, published after submission of my manuscript, Nietfeld and colleagues report an estimated (not actual) lifetime risk of needing haematopoietic stem cell transplantation of 1:200 and, for autologous transplantation, a risk of 1:400 (REF. 4). However, many assumptions in this paper have yet to be questioned and the data is based on an estimated, not actual, transplant population.

In a further recent report ${ }^{5}$, Nietfeld et al. have responded to the American Society of Blood and Marrow Transplantation 2008 Position Statement on the Collection and Preservation of Cord Blood for Personal Use $^{6}$ questioning the previous estimated probability of needing an autologous cord blood transplant. In doing so they have erroneously linked the risk of needing haematopoietic stem cell transplantation (HSCT) with the probability of using one's own cord blood for an autologous transplant. These are not one and the same; however, one can assume that commercial cord banks will use this prediction to justify continued commercial cord banking.

In analysing the chance of needing an autologous HSCT in the first 20 years of life, I have not restricted my analysis to the $0-14$ age group, as stated by Nietfeld, but rather looked at indications for each specific disease diagnosis (see Table 1 in REF. 1). However, as it so happens, most of conditions benefiting from autologous stem cell transplant under the age of 20 occur in younger children.

Here, and in his paper, Nietfeld includes both brain tumours and sarcomas as indications for autologous stem cell transplantation. However, this assumption is flawed. There is no compelling evidence that high-dose therapy with autologous HSCT improves the outcome for the common malignant brain tumours, such as high-grade gliomas or glioblastoma, in either adults or children ${ }^{7,8}$. Similarly, autologous HSCT has no role in the routine treatment of standard or high-risk medulloblastoma, or ependymoma in older children ${ }^{9}$. Autologous HSCT for brain tumours in those under the age of 20 is largely restricted (with some exceptions) to younger children with highrisk medulloblastoma $(<10)$ as part of open collaborative clinical trials (Headstart III and COG ACNS0334) ${ }^{10,11}$. However, many contemporary clinical trials use tandem procedures with multiple cycles of autologous HSCT, which is not possible with a single stored unit of cord blood. The outcome of these studies is pending completion of the trials. Some rare but chemosensitive brian tumours in children, such as germinoma and pineoblastoma, may respond to high-dose therapy and autologous HSCT ${ }^{12}$.

Similarly, with regard to sarcomas, and again contrary to Nietfeld's claim, autologous HSCT has no role in the treatment of primary localized or metastatic soft tissue sarcomas, rhabdomyoscarcoma or osteosarcoma in either children or adults ${ }^{13-18}$. Autologous transplantation has a limited role in the treatment of some recurrent sarcomas and of metastatic Ewing's sarcoma (pulmonary metastases only) as part of the collaborative European and North American clinical trials (EuroEwing 99 and COG AEWS0331 $)^{15}$. Again these trials remain open for accrual and results will not be published for several years.

However, as I stated previously, there is a role for autologous HSCT in other tumour groups such as relapsed lymphoma and highrisk metastatic testicular germ cell tumours, and a more limited role in rare instances of chemotherapy-sensitive relapsed solid tumours such as metastatic Wilms tumour ${ }^{1}$.

The common error made by Nietfeld and others in estimating the likelihood of use' for autologous cord blood is failing to consider that in nearly all cancers where an autologous HSCT is indicated, other sources of haematopoietic stem cells are readily available at the time the cancer is diagnosed, either from the bone marrow or by harvest of peripheral blood stem cells, obviating the need for autologous cord blood storage ${ }^{1}$.

Hence, autologous cord blood is only indicated as an alternative source of stem cells for HSCT in diseases that have a proven chance of cure and where a standard stem harvest is not possible, either because the bone marrow is itself involved with disease or because there is acquired marrow failure syndrome such as aplastic anaemia. Nietfeld also omitted to consider that autologous cord blood cannot not be used for contemporary intense tandem autologous stem cell transplants in cancers.

It is not possible to make an accurate estimate of the chances of using an autologous cord blood sample given the rarity of the cancers and the uniqueness of many clinical indications. However, Nietfeld's own estimate of needing an autologous stem cell transplant in the first 20 years, excluding brain tumours and sarcomas as noted above, is $0.0092 \%(\sim 1: 11,000)$ and, given that most of these cases will be treatable without cord blood, I stand by my statement that the chance of using stored autologous cord blood is substantially less and indeed unlikely.

Both Harris and Nietfeld refer to recent clinical trials using autologous cord blood in non-malignant disorders, in particular type I diabetes and neonatal hypoxic ischaemic encephalopathy and cerebral palsy, as evidence to support the autologous storage of cord blood. The University of Florida study of type I diabetes (NCT00305344) is a phase I/II study recruiting a total of 23 eligible children with available autologous cord blood. The hypothesis is that autologous cord blood may modulate the host immune response and that endogenous cord-derived stem cells may contribute to pancreatic tissue repair. This is a non-randomized study with no control group. Ironically, the $a$ priori animal data supporting the study were based on bone marrow-derived mesenchymal stem cells in mice, not those from cord blood (NCT00305344). The Duke study of hypoxic-ischaemic encephalopathy (NCT00593242) is a phase I, non-randomized, historical control study recruiting a total of 12 eligible newborns to determine the safety of autologous cord blood reinfusion in newborns with clinical signs of acute hypoxic-ischaemic encephalopathy. The 
secondary endpoints will be the assessment of neurodevelopmental outcome and measurable changes on neuroimaging compared with normal and historic controls. The outcome of this study will be awaited with interest, as cord blood taken at birth is haematologically indistinguishable from the newborn's own circulating blood and in neonatal terms is simply a top-up transfusion! An additional Duke study is enrolling patients with established cerebral palsy for treatment with autologous cord blood, but evidence for benefit of this therapy will depend on the outcome of controlled, randomized studies comparing autologous cord blood transplantation with other therapies, especially the use of other sources of stem cells such as bone marrow and peripheral blood.

Harris has raised the issue of family banking, in which storage of cord blood may benefit a relative such as a sibling or parent. Such storage is termed 'directed' when there is a known genetic disease risk or affected at-risk individual, and 'nondirected' when there is no known genetic risk or affected family member. Directed cord blood storage is clearly indicated for expectant parents who already have a child with a cancer or genetic disorder treatable by an allogeneic HSCT $^{6}$. Most public cord blood banks offer directed cord blood storage without a fee and, in the United States, the National Institutes for Health fund the Oakland Directed Cord Blood Program for the treatment of haemoglobinopathies and malignancy ${ }^{6}$.

However, non-directed or family banking, where there is no at-risk individual, is currently not recommended, as the chance of use in the absence of a high-risk factor is low ${ }^{6}$.

The pluripotency of cord blood-derived stem cells raised by Harris remains a moot point at present, particularly given the recent challenge to the notion of 'stemness' arising from work on the induction of pluripotency in differentiated adult tissues ${ }^{19-23}$.

Indeed, in a recent phenotypical analysis of multiple human stem cell lines, Muller et al. used microarray gene expression analysis and a machine learning algorithm to classify 150 pluripotent, multipotent and adult stem cell lines, including the recently defined differentiated human umbilical vein endothelial stem cells ${ }^{24}$.

Pluripotent stem cells cluster as a discrete subset of cell lines expressing a unique profile of stem cell-related proteins (PluriNet), which influence stem cell growth, self-renewal and differentiation. However, haematopoietic and mesenchymal multipotent stem cells and the human umbilical vein endothelial stem cells did not cluster in the pluripotent profile.

However, of crucial importance for the future direction of stem cell-based therapies, induced pluripotent stem cells (iPS), which are derived from adult differentiated tissues, cluster with pluripotency, supporting the notion that pluripotency can be indeed be derived from differentiated adult tissues ${ }^{24}$.

Finally, it appears that direct reprogramming of differentiated adult cells is now possible even without the induction of pluripotency. Zhou et al. used a viral transfection strategy to re-express developmentally regulated genes in the adult mouse pancreas and, in vivo, have reprogrammed adult mouse exocrine cells into $\beta$-islet cells. Molecular analysis showed these induced cells are phenotypically and functionally indistinguishable from endogenous mouse $\beta$-islet cells ${ }^{25}$.

Finally, both Harris and Nietfeld justify commercial cord blood banking by observing that over 60 autologous cord blood transplants have been done to date (http://www.parentsguidetocordblood. org). However, of these 60 cases, most were enrolled recently in the Duke and Florida trials for hypoxic encephalopathy and cerebral palsy, and diabetes, respectively. Only 17 cases were treated for malignancy (11) or acquired aplastic anaemia (6). A recent survey of US commercial cord blood banks by the American Society for Blood and Marrow Transplantation reported a total of 490,000 privately banked cord blood units ${ }^{6}$. At an average of US $\$ 2,000$ to collect and store a cord blood unit for 20 years, the US health consumer has invested nearly US $\$ 1$ billion, resulting in the treatment of just 17 patients with malignancy or marrow failure and with no peer-reviewed published clinical outcome data.

It remains clear that marketing commercial cord banking to expectant parents for the treatment of diseases by autologous HSCT cannot currently be justified, and claims for the future use of cord blood for regenerative medicine need to be reevaluated in the light of recent technical developments in stem cell technology.

Michael J. Sullivan is at the Children's Cancer Research Group, University of Otago, Christchurch, 2 Riccarton Road, PB 4,710, Christchurch, New Zealand. e-mail:michael.sullivan@otago.ac.nz

Sullivan, M. J. Banking on cord blood stem cells. Nature Rev. Cancer 8, 555-563 (2008).

2. Nietfeld, J. J. Opinions regarding cord blood use need an update. Nature Rev. Cancer 24 Sep 2008 (doi:10.1038/nrg2418-c1)

3. Harris, D. T. Cord blood stem cells: worth the investment. Nature Rev. Cancer 24 Sep 2008 (doi: 10.1038/nrg2418-c2).
$4 \quad$ Nietfeld, J. J., Pasquini, M. C., Logan, B. R., Verter, F. \& Horowitz, M. M. Lifetime probabilities of hematopoietic stem cell transplantation in the US Biol. Blood Marrow Transplant. 14, 316-322 (2008).

5. Nietfeld, J. J., Pasquini, M. P., Logan, B. R., Verter, F. \& Horowitz, M. M. On the probability of using cord blood. Biol. Blood Marrow Transplant. 14, 724-725 (2008).

6. Ballen, K. K., Barker, J. N., Stewart, S. K., Greene, M. F. \& Lane TA; American Society of Blood and Marrow Transplantation. Collection and preservation of cord blood for personal use. Biol. Blood Marrow Transplant. 14, 356-363 (2008).

7. Durando, X. et al. High-dose BCNU followed by autologous hematopoietic stem cell transplantation in supratentorial high-grade malignant gliomas: a retrospective analysis of 114 patients. Bone Marrow Transplant. 31, 559-564 (2003).

8. Jacques-Olivier, B. et al. Does high-dose carmustine increase overall survival in supratentorial high-grade malignant glioma? An EBMT retrospective study. Int. J. Cancer 120, 1782-1786 (2007).

9. Zacharoulis, S. et al. Outcome for young children newly diagnosed with ependymoma, treated with intensive induction chemotherapy followed by myeloablative chemotherapy and autologous stem cell rescue. Pediatr. Blood Cancer 49, 34-40 (2007).

10. Thorarinsdottir, H. K. et al. Outcome for children $<4$ years of age with malignant central nervous system tumors treated with high-dose chemotherapy and autologous stem cell rescue. Pediatr. Blood Cancer 48, 278-284 (2007).

11. Guruangan, S. et al. Myeloablative chemotherapy with autologous bone marrow rescue in young children with recurrent malignant brain tumors J. Clin. Oncol. 16, 2486-2493 (1998).

12. Gururangan, S. et al. High-dose chemotherapy with autologous stem-cell rescue in children and adults with newly diagnosed pineoblastomas. J. Clin. Oncol. 21, 2187-2191 (2003)

13. Yamada, K. et al. High-dose chemotherapy and autologous peripheral blood stem cell transfusion for adult and adolescent patients with small round cell sarcomas. Bone Marrow Transplant. 39, 471-476 (2007).

14. Ek, E. T. H. \& Choong, P. F. M. The role of high-dose therapy and autologous stem cell transplantation for pediatric bone and soft tissue sarcomas. Exp. Rev. Anticancer Ther. 6, 225-237 (2006).

15. Meyers, P. A. High-dose therapy with autologous stem cell rescue for pediatric sarcomas. Curr. Opin. Oncol. 16, 120-125 (2004)

16. Schlemmer, M. et al. Efficacy of consolidation highdose chemotherapy with ifosfamide, carboplatin and etoposide (HD-ICE) followed by autologous peripheral blood stem cell rescue in chemosensitive patients with metastatic soft tissue sarcomas. Oncology 71, 32-39 (2006).

17. Boulad, F. et al. High-dose induction chemoradiotherapy followed by autologous bone marrow transplantation as consolidation therapy in rhabdomyosarcoma, extraosseous Ewing's sarcoma, and undifferentiated sarcoma. J. Clin. Oncol. 16, 1697-1706 (1998).

18. Koscielniak, E. et al. Do patients with metastatic and recurrent rhabdomyosarcoma benefit from high-dose therapy with hematopoietic rescue? Report of the German/Austrian Pediatric Bone Marrow Transplantation Group. Bone Marrow Transplant. 19, 227-231 (1997)

19. Maherali, N. et al. Directly reprogrammed fibroblasts show global epigenetic remodeling and widespread tissue contribution. Cell Stem Cell 1, 55-70 (2007).

20. Park, I. H. et al. Reprogramming of human somatic cells to pluripotency with defined factors. Nature 451, 141-146 (2008)

21. Wernig, M. et al. In vitro reprogramming of fibroblasts into a pluripotent ES-cell-like state. [see comment]. Nature 448, 318-324 (2007).

22. Yamanaka, S. Strategies and new developments in the generation of patient-specific pluripotent stem cells. Cell Stem Cell 1, 39-49 (2007).

23. Yu, J. et al. Induced pluripotent stem cell lines derived from human somatic cells. Science 318, 1917-1920 (2007).

24. Müller, F.-J. et al. Regulatory networks define phenotypic classes of human stem cell lines. Nature 27 Aug 2008 (doi: 10.1038/nature07213).

25. Zhou, Q., Brown, J., Kanarek, A., Rajagopal, J. \& Melton D. A. In vivo reprogramming of adult pancreatic exocrine cells to $\beta$-cells. Nature 27 Aug 2008 (doi: 10.1038/nature07314). 